\title{
Argatroban Anhydrous
}

National Cancer Institute

\section{Source}

National Cancer Institute. Argatroban Anhydrous. NCI Thesaurus. Code C75153.

The anhydrous form of argatroban, a synthetic derivative of L-arginine with

antithrombotic activity. Argatroban is a univalent and direct inhibitor of fibrin-bound

thrombin. This agent reversibly binds to the thrombin active site, thereby preventing the thrombin-dependent reactions, which include the conversion of fibrinogen to fibrin; the activation of factors V, VIII and Xl; the activation of protein C; and platelet aggregation. Argatroban is highly selective for thrombin and is able to inhibit the action of both free and clot-associated thrombin. As a result, stabilization of blood clots occurs and coagulation is inhibited. 